\title{
An unusual reason of abdominal pain: Rapunzel syndrome case report and literature review
}

\author{
Sevgi Buyukbese Sarsu ${ }^{1 *}$, Ali Karapur ${ }^{1}$
}

\begin{abstract}
Bezoar is defined as a mass formed as a consequence of the accumulation of indigestible foreign substances within the gastric outlet or small bowels with resultant dysfunctional gastric emptying Gastric bezoar extending into the small or large bowel and causing a clinical condition called Rapunzel syndrome This rare pediatric condition is observed in young girls with psychiatric problems such as trichotilomania, who frequently feel an irresistable desire to pluck out, and swallow their hairs. A 12-year-old girl was admitted to our hospital with complaints of abdominal pain, growth retardation, and partial hair loss. Endoscopic examination revealed the presence of gastroduodenale trichobezoar, and her giant trichobezoar was extracted using conventional laparotomy, and anterior gastrostomy. With this case representation we reported a case with Rapunzel Syndrome in the light of literature data.
\end{abstract}

Key words: Unusual abdominal pain, gastroduodenal trichobezoar, trichotillomania, abdominal mass, growth retardation, laparotomy, child

\section{Introduction}

Bezoars are defined as masses formed by indigestible materials which aggregate, solidify in the stomach and bowels of humans after the oral intake. Bezoar is a word derived from Persian (Persian pad-zahr, from pad ) which means antidote. In the literature, formation of bezoars from drugs, sand particles, chewing gum, mushrooms, antacids, sponge, and sunflower seeds have been reported $(1,2)$. They are classified based on the content. For instance, continuous intake of plastic material (plastobezoar), antacid-like drugs (pharmobezoar), vegetable, and fruit -based materials (phytobezoar), milk and milk products (lactobezoar) and plucked hairs (trichobezoar) cause development of various kinds of bezoars indicated in the brackets.

Trichobezoar is a rarely encountered condition emerging with symptoms and signs generally involving the gastrointestinal system. Human hair can easily accumulate between gastric mucosal folds because of its smooth surface. Ingested food particles and gastric mucus together with human hair can form a trichobezoar (2). Trichobezoars can be localized only in the stomach or they can extend into pylorus, duodenum, jejunum, and colon.

Fragments broken from the terminal part of the trichobezoar which is localized in the proximal part of the stomach or small bowel can cause intestinal obstruction termed as Rapunzel syndrome. (2).

It was firstly reported in the literature in 1779 as an autopsy finding in a patient who died due to peritonitis development as a consequence of gastric perforation. This clinical condition was named after a 12 -yearold, long-haired fairy tale character Rapunzel.The first use of the term was recorded in 1968 by Vaughan et al. to define a case with trichobezoar (3-6). Since it manifests itself with nonspecific symptoms of intraabdominal mass, abdominal pain, weight loss, nausea and vomiting, constipation or diarrhea. This syndrome generally cannot be diagnosed at its early stage. Most of the time, underlying mental or psychiatric diseases accompany this condition (2).

Cases of mucosal ulcer, and perforation, proteinenergy malnutrition, obstructive jaundice, pancreatitis and death related to trichobezoar have been reported (2). Even though laparoscopic and endoscopic methods have been used in the treatment, most of the time, because of the presence of bulky masses, conventional laparotomy has been preferred $(2,3)$. In this case, report diagnosis and treatment approach in a female child with rarely encountered Rapunzel syndrome has been reported in the light of the literature. 


\section{Case}

A 12-year-old girl was referred to the outpatient clinics of Pediatric Surgery of Cengiz Gokcek Obstetrics and Children's Hospital with complaints of growth retardation persisting for nearly a year. It was determined from patient's anamnesis that her abdominal pain was of colicky type and localized on the epigastric region. She had not had any known disease or undergone surgery previously. Her family history revealed that she had lost her parents at an early age and brought up by her second-degree relatives. On physical examination, her general health state was in good condition. She was $136 \mathrm{~cm}(<3 \%)$ tall, and weighed $30.5 \mathrm{~kg}(<3 \%)$. She had partial alopecia. On abdominal palpitation, a solid, mobile mass with irregular contours was detected over epigastric region. Laboratory tests did not reveal any pathology apart from iron deficiency- anaemia. Standing, plain abdominal radiograms demonstrated an opacity localized in the left upper abdominal quadrant. Accordingly, on EGD examinations performed, a giant mass completely filling the stomach and extending into the duodenum was detected. On abdominal CT, over distended stomach, and an image extending from the gastric lumen into duodenum which suggested the presence of a foreign body was observed. Esophagogastroduodenoscopy, the fixed mass extending from gastric cardia into duodenum, which did not allow passage of the endoscope was evaluated as trichobezoar. Since the mass was very bulky, which was not amenable to endoscopic extraction, and its surface was too hard to be fragmented with a biopsy forceps through supraumbilical midline laparotomy incision exploratory surgery was started. Anterior gastrostomy incision was performed on gastric body, and a trichobezoar which completely filled the stomach which then extended into the second part of the duodenum, and took its shape was extracted (Figures 1 , and 2).

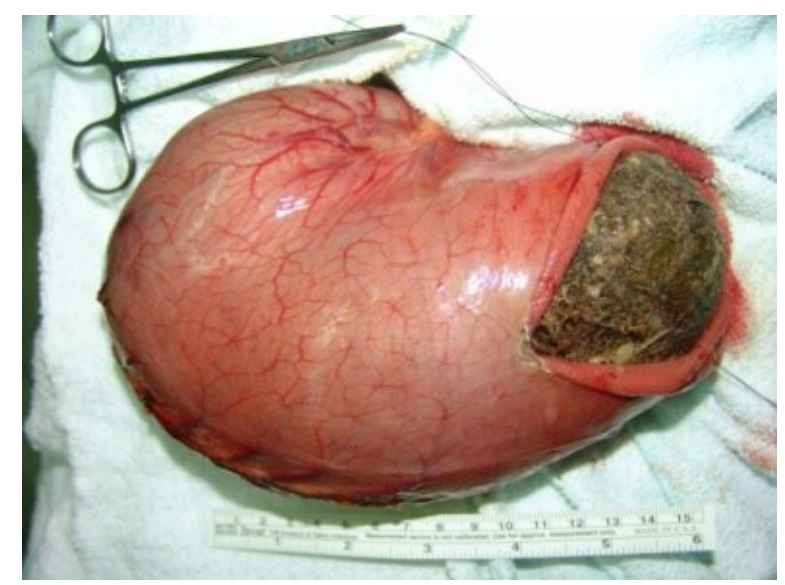

Figure 1: Anterior gastrotomy incision on the gastric body

Medical Science and Discovery 2016; 3(2): 112-5

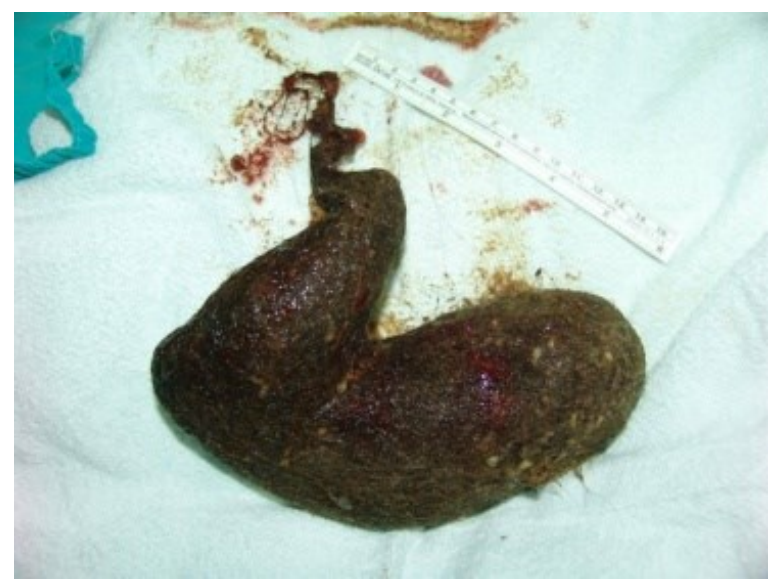

Figure 2: Trichobezoar which took the shape of the stomach, and the duodenum

In the histopathological examination, a hairball which contained food residues and took the shape of the stomach measuring $32 \times 11 \mathrm{~cm}$ and weighing $1.8 \mathrm{~kg}$ was detected. The patient did not experience any complications during the postoperative period, therefore she was discharged on the 7th postsurgery day. The pediatric psychiatry detected obsessivecompulsive disorder and trichotillomania (impulsive hair plucking) with recommendation of ambulatory controls. Enlightened consent form was obtained from the patient

\section{Discussion}

Trichobezoar is a type of bezoar which can generate unusual, abdominal pain in childhood and adolescence. Since the mass grows slowly and yields nonspecific symptoms, it generally remains concealed for a long time. Symptoms like abdominal pain, nausea, vomiting, weakness, growth retardation, alopecia, history of psychiatric disease, nutritional, and vitamin deficiencies, iron deficiency anemia, palpable epigastric mass (Lamerton sign), detection of hair strands in stool in addition to radiological findings aid in the diagnosis of trichobezoars (2). In our case, colicky abdominal pain, growth retardation, and partial alopecia were found. Especially contrastenhanced radiograms can be helpful in the differential diagnosis of the masses localized in the upper gastrointestinal system. Endoscopy is known to have the highest sensitivity and specificity because it provides information about the structure of the mass. On ultrasonograms, they can be seen as a wide hyperechoeic band localized in the stomach, and bowels. CT examination can yield information about the location, and distribution characteristics of the bezoar. In our case, even if a mass lesion was detected in EGD examinations and abdominal $\mathrm{CT}$, the diagnosis could not be confirmed till GIS endoscopy was applied. In the literature, it has been indicated that a case of trichobezoar which cannot be detected in the 
short-term can cause ileus, perforation, ulceration, bleeding, pancreatitis, obstructive jaundice, superior mesenteric artery syndrome, invagination, peritonitis or even death (3).

Continuous intake of indigestible materials is mostly held responsible from development of trichobezoar (2). The etiological factos include psychiatric problems including underlying depression, anxiety, obsessive compulsive disorder, emoitional factors such as stress, problematic family environment, loss of mother and/or father or mental retardation. Our patient had lost her parents. Generally, the underlying cause of trichobezoars is an impulse control disorder named trichotillomania. Trichotillomania which was firstly described in 1889 , is a condition of partial alopecia (partial baldness), mainly caused by impulsive hair pluckings. Patchy areas of baldness were also detected in our case. Some authors have also reported that these patients had felt a desire even to eat their eye brows, eyelashes, arm, leg, and pubic hair. The incidence of trichotillomania ranges between 0.6 , and $1.6 \%$, and in $30 \%$ of these patients.

Although trichobezoars are known to be most prevalent in the age of 13-20 years, a 54-year-old case has been reported in the literature. They are most frequently localized in the stomach and take the shape of this organ in the long-term. Their migration from the stomach into the small bowel may cause obstructive jaundice, pancreatitis, protein-loss enteropathy (7). Giant trichobezoars develops only in one percent of the cases. Our case was quiet a large trichobezoar in a small girl. In "Rapunzel syndrome," the terminal end of trichobezoar localized in the stomach extends towards small bowel. This condition is a very rarely seen form of trichobezoar, and more than $90 \%$ of the cases consist of girls younger than 20 years of age $(2,4)$. In our case, a trichobezoar completely filling the stomach of a 12-year-old girl, and extending into the duodenum was observed.

Phytobezoar is the most prevalent type of bezoar which is usually encountered in adult patients secondary to gastric motility, and consequently decrease in gastric acid levels. Risk factors for the development of phytobezoar include history of gastric surgery like vagotomy, pyloroplasty, Billroth II and gastroenterostomy. In the pathogenesis of phytobezoar formation in these patients include decrease in truncal gastric acidity and delayed gastric emptying. More rarely, it can be seen in cystic fibrosis patients who had lung transplantation or following cholecystectomies (2). However, lactobezoars can develop during neonatal period secondary to use of concentrated baby formulas for premature infants.

The objectives of the treatment include extraction of the mass as a whole and prevention of recurrences. Very small bezoars at the time of diagnosis can be thrown out naturally through gastrointestinal route by using a liquid diet and prokinetic drugs (2). In the endoscopic treatment, bezoar mass is fragmented into small pieces using biopsy forceps before the extraction. Success rates of this method ranges between 85-90 percent (2). Use of extracorporal shock waves or Nd-YAG laser for the fragmentation of the bezoar is already acknowledged $(2,8)$. Apart from endoscopic fragmentation, application of intragastric enzymes (cellulose, lipase, and $\mathrm{N}$-acetylcysteine) enables disintegration of bezoars (8). Phytobezoars and lactobeozoars are relatively small and can easily be broken into pieces, so endoscopic treatment is effective in their fragmentation. However, in trichobezoars the success rates of endoscopic treatments are not so great.

Since hairs contained in trichobezoars are resistant to mechanical fragmentation or enzymatic disintegration, generally surgical treatment is preferred instead of endoscopic interventions. Besides, emergence of complications caused by bezoars creates an indication for surgical interventions (9). In this case, open or laparoscopic surgery can be used. In the laparoscopic method, broken fragments of trichobezoars can cause intestinal occlusion and hair strands falling into the peritoneal space may result in complications. In the Rapunzel syndrome, currently open surgery and anterior gastrotomy are recommended so as to extract giant gastric bezoars (8). Success rate of $99 \%$ has been reported for conventional surgery (3). In this case, we made a diagnosis of trichobezoar based on endoscopic examination, and then we performed conventional laparotomy. Bezoar can sometimes simultaneously occur in more than one foci, so complete gastrointestinal system exploration should be made. In our patient, since the hair ball was very bulky we extracted it with open surgery and complete intestinal exploration was performed. Some authors reported development of recurrences following surgical interventions $(3,10)$. During our two years of follow-up, we haven't encountered recurrences related to the presence of bezoar.

Trichobezoar is an important condition which should be treated before the development of complications. Therefore, in young girls complaining of abdominal pain and abdominal mass who have psychiatric diseases accompanied with habits of hair plucking (trichotillomania) in addition of trichophagia (eating hairs) possibility of trichobezoar should be contemplated. In its treatment, still conventional laparotomy is used prevalently. Besides, as an important issue, young girls with trichobezoars should be also followed up by pediatric psychiatry in order to prevent development of recurrences.

Conflict of Interest: The authors declare no potential conflicts of interest with respect to the research, authorship, and/or publication of this article.

Medical Science and Discovery 2016; 3(2): 112-5 


\section{References}

1. Lopes LR, Oliveira PSP, Pracucho EM, Camargo MA, de Souza Coelho Neto J, Andreollo NA. The rapunzel syndrome: an unusua trichobezoar presentation. Case Report Med 2010; 2010: 841028.

2. Kırkıl C, Böyük A, Bülbüller N, Aygen E, Karabulut K. Nadir rastlanan bir trikobezoar olgusu: Rapunzel sendromu. Gaziantep Tip Derg 2011; 17(2): 108-110.

3. Gorter RR, Kneepkens CM, Mattens EC, Aronson DC, Heij HA. Management of trichobezoar: case report and literature review. Pediatr Surg Int 2010; 26: 457-63.

4. Vila S, Garcia C, Piscoya A. Giant gastroduodenal trichobezoar Rapunzel syndrome. Am J Gastroenterol 2009; 104: 2864-5.

5. Crawley AJ, Guillerman RP. Rapunzel syndrome. Pediatr Radiol 2010; 40: S100.
6. Bashir EA, Samiullah, Sadiq MA, Yusuf O, Karim K. Rapunzel syndrome. J Ayub Med Coll Abbottabad 2010; 22: 218-20.

7. Chogle A, Bonilla S, Browne M, Madonna MB, Parsons W, Donaldson J, Alonso E.Rapunzel syndrome: a rare cause of biliary obstruction. J Pediatr Gastroenterol Nutr 2010; 51: 522-3.

8. Sharma D, Srivastava M, Babu R, Anand R, Rohtagi A, Thomas S. Laparoscopic treatment of gastric bezoar. JSLS 2010; 14: 263-7.

9. Hernandez-Peredo-Rezk G, Escarcega-Fujigaki P, CampilloOjeda ZV, Sanchez-Martinez ME, Rodriguez-Santiba-ez MA, Angel- Aguilar AD, et al. Trichobezoar can be treated laparoscopically. J Laparoendoscop Adv Surg Tech A 2009; 19: 111-3.

10. Tiwary SK, Kumar S, Khanna R, Khanna AK. Recurrent rapunzel syndrome. Singapore Med J 2011; 52(6): 128-30.

Copyright (C) 2014 The Author(s); This is an open-access article distributed under the terms of the Creative Commons Attribution License (http://creativecommons.org/licenses/by/4.0), which permits unrestricted use, distribution, and reproduction in any medium, provided the original work is properly cited. All Rights reserved by international journal of Medical Science and Discovery.

Medical Science and Discovery 2016; 3(2): 112-5 